\title{
Cardiovascular disease risk among Chinese antiretroviral-naïve adults with advanced HIV disease
}

\author{
Fuping Guo ${ }^{1 \dagger}$, Evelyn Hsieh ${ }^{1,2 \dagger}$, Wei Lv${ }^{1}$, Yang Han ${ }^{1}$, Jing Xie ${ }^{1}$, Yanling $\mathrm{Li}^{1}$, Xiaojing Song ${ }^{1}$ and Taisheng Li ${ }^{*}$
}

\begin{abstract}
Background: Cardiovascular disease (CVD) is an important cause of mortality among HIV-infected patients, however little is known about the burden of CVD among this population in Asia. We sought to quantify prevalence of CVD risk factors, 10-year CVD risk, and patterns of CVD risk factor treatment in a group of individuals with HIV in China.

Methods: We retrospectively analyzed baseline data from treatment-naïve HIV-infected adults enrolled in two multicenter clinical trials in China. Data regarding CVD risk factors such as smoking, hypertension, diabetes, dyslipidemia and obesity were assessed. The Framingham Risk Score (FRS) and Data Collection on Adverse Events of Anti-HIV Drugs (D:A:D) risk scores were calculated to estimate 10-year CVD risk. The American College of Cardiology/American Heart Association Atherosclerotic Cardiovascular Disease (ASCVD) Risk Score was used to identify individuals meeting criteria for lipid-lowering therapy.
\end{abstract}

Results: In total, 973 patients were included in the analysis. Mean age was $36.0 \pm 10.2$ years and $74.2 \%$ were men. The most common CVD risk factors were dyslipidemia (51.7\%) and smoking (23.7\%). Prevalence of hypertension, diabetes and obesity were $8.4 \%, 4.6 \%$ and $1.0 \%$, respectively. Over $65 \%$ of patients had at least one CVD risk factor. The prevalence of 10 -year risk of CVD $\geq 10 \%$ was $4.5 \%$ based upon FRS and was $3.3 \%$ based upon D:A:D risk score. Few patients with dyslipidemia, hypertension or diabetes were on treatment.

Conclusions: CVD risk factors are common but under-treated among Chinese treatment-naïve individuals with HIV. Future interventions should focus on training HIV providers to appropriately recognize and manage CVD risk factors during routine clinical assessments.

Keywords: HIV, Cardiovascular disease risk, Framingham risk score, Data collection on adverse events of anti-HIV drugs (D:A:D) risk score, Atherosclerotic cardiovascular disease risk score

\section{Background}

Increasing access to antiretroviral therapy (ART) has ostensibly changed HIV infection in many parts of the world from a fatal diagnosis to a chronic condition requiring lifelong monitoring and treatment. However, this extended life expectancy comes with unique long-term complications. Prior studies, largely from the USA and Europe, have demonstrated that cardiovascular disease (CVD) is a common cause of morbidity and mortality

\footnotetext{
* Correspondence: litsh@263.net

'Equal contributors

${ }^{1}$ Department of Infectious Diseases, Peking Union Medical College Hospital,

Chinese Academy of Medical Sciences, Beijing, China

Full list of author information is available at the end of the article
}

among individuals with HIV, and the second cause of non-AIDS related deaths (1.6 per 1000 person-years) after liver disease $[1,2]$. Causes for the increased CVD risk observed among HIV-infected patients include both traditional risk factors, including aging, higher smoking rates, dyslipidemia, insulin resistance, deposition of body fat; and non-traditional factors, including inflammation, direct effects of HIV on the vasculature, and toxicity from ART $[3,4]$.

Several Western studies have demonstrated an increased risk of CVD among HIV-infected patients compared with HIV-negative populations using validated tools such as the Framingham CVD Risk Score (FRS), Data Collection 
on Adverse events of Anti-HIV Drugs (D:A:D) risk scores and the American College of Cardiology/American Heart Association (ACC/AHA) Atherosclerotic Cardiovascular Disease Risk Score (ASCVD) [4-8]. However, few studies have focused on CVD risk assessment among individuals with HIV in Asia. Two studies from Thailand found the prevalence of predicted cardiovascular risk in HIVinfected Thai patients was relatively low $[9,10]$. In China, the prevalence of risk factors for CVD is high in the general population [11, 12], and CVD has become the leading cause of morbidity and mortality in recent decades [13]. No studies to date have assessed the underlying prevalence of CVD risk factors among Chinese individuals with HIV prior to initiation of ART.

To address this gap, we compiled baseline data from two large multicenter studies of individuals with HIV across China from 2009 to 2012, and measured the prevalence of CVD risk factors, 10-year CVD risk, and patterns of CVD risk factor treatment prior to initiation of ART.

\section{Methods}

Study design

We performed a retrospective cross-sectional analysis of baseline data collected as part of two large multicenter clinical trials from 2009 to 2012 across 11 provinces and municipalities of China that are regions of high HIV prevalence, including Beijing, Fujian, Guangdong, Guangxi, Henan, Hunan, Liaoning, Shanghai, Shanxi, Sichuan, and Yunnan province. The patients therefore represented a broad cross-section of the overall population of HIVinfected patients in China. The details of these clinical trials have been previously described $[14,15]$.

Data from all participants enrolled in the two multicenter clinical trials were considered for inclusion in this analysis. All participants were adults aged 18-65 years, with documented HIV infection confirmed by Western blot analyses, CD4+ cell count $<500 \mathrm{cells} / \mathrm{mm}^{3}$, and antiretroviral-naive. From a total of 1607 patients enrolled in the two parent studies, 973 patients (61\%) had complete data regarding CVD risk factors and were included in the present analysis.

\section{Measurements}

For each parent study, detailed sociodemographic and clinical data were collected by trained study staff at the baseline encounter and recorded in electronic research databases $[13,14]$. Fasting plasma samples were collected as part of routine screening for HIV care at each study site, and results were subsequently entered into the electronic study database as part of the parent study protocols.

\section{Sociodemographic and clinical characteristics}

Data for the following sociodemographic and clinical variables were analyzed: age, sex, height, weight, body mass index (BMI), route of HIV transmission, years since HIV diagnosis, medical co-morbidities, and medication history. Data regarding smoking history was collected for each participant in the parent trials and included current smoking (yes/no) as well as frequency of smoking and number of cigarettes per day. BMI was further classified according to the guidelines established by the United States National Institutes of Health National Heart, Lung, and Blood Institute, with the following categorizations: underweight $<18.5$, normal 18.5-24.9, overweight 25-29.9, and obese $\geq 30.0 \mathrm{~kg} / \mathrm{m}^{2}[16]$.

Blood pressure $(\mathrm{mmHg})$ was measured using a manual sphygmomanometer on the patient's arm while the patient was sitting at rest. Hypertension was defined based upon the Joint National Committee on Prevention, Detection, Evaluation and Treatment of High Blood pressure recommendations [17]. Patients with a systolic blood pressure (SBP) of at least $140 \mathrm{mmHg}$ or diastolic blood pressure (DBP) of at least $90 \mathrm{mmHg}$ on two or more visits, and patients prescribed antihypertensive medication were considered to have hypertension.

Dyslipidemia was defined as total cholesterol (TC) $>5.2 \mathrm{mmol} / \mathrm{l}$, high-density lipoprotein cholesterol (HDL-c) $<1.0 \mathrm{mmol} / \mathrm{l}$, low-density lipoprotein cholesterol (LDL-c) $>4.1 \mathrm{mmol} / \mathrm{l}$, or triglycerides (TG) $>1.7 \mathrm{mmol} / \mathrm{l}$ based upon the United States National Cholesterol Education Program, Adult Treatment Panel (NCEP-ATP) III guidelines [18].

Patients were classified with diabetes if they had a prior diagnosis, a fasting plasma glucose $\geq 7.0 \mathrm{mmol} / \mathrm{l}$ or were being treated with insulin or oral hypoglycemic agents [10].

\section{Laboratory analyses}

For the purposes of this study we collected the following laboratory data from the two parent clinical trial databases: fasting plasma TC, HDL-c, TG, LDL-c, and glucose levels. As well plasma CD4+ cell counts and HIV-1 RNA viral load data were collected. The laboratory techniques used to obtain these measurements have been described previously $[13,14]$.

\section{Cardiovascular disease risk classification}

We employed three CVD risk prediction models including the FRS, D:A:D risk scores and the ACC/AHA ASCVD Risk Score. For individuals $\geq 20$ years of age, the FRS calculates 10-year predicted risk of CVD based upon a model comprised of age, sex, TC, HDL-c, SBP, current treatment for hypertension and cigarette smoking (anytime in the past month) [18]. The CVD risk prediction model also categorizes individuals as low $(<10 \%$ 10 -year risk), intermediate (10-20\% risk) or high risk $(\geq 20 \%$ risk) for coronary heart disease $[3,19,20]$.

D:A:D 10-year estimated CVD risk scores were calculated based upon age, gender, current smoking, exsmoking status, total and HDL cholesterol, systolic blood 
pressure, diabetes, family history of CVD and prior use of specific ART $[4,21]$. Patients in our study were ART-naïve at enrollment and had no known family history of CVD. Therefore, the value for covariates in the algorithm representing specific ART drugs conferring CVD risk (duration of indinavir, lopinavir, abacavir) were set to zero and no prior family history of CVD was assumed in the calculation of the D:A:D risk prediction equations as has been described previously [10].

For individuals $\geq 40$ years of age, we also calculated the ACC/AHA ASCVD Risk Score based upon age, sex, race, total cholesterol, HDL-c, SBP, current treatment for hypertension, diabetes and current smoking (yes/ no) (http://tools.acc.org/ASCVD-Risk-Estimator/). This score estimates a 10-year risk for ASCVD for patients aged 40 to 79 years.

We further estimated the proportion of individuals with a favorable CVD risk profile, which was defined on the basis of a number of modifiable risk factors as follows: total cholesterol $<5.17 \mathrm{mmol} / \mathrm{l}, \mathrm{SBP} \leq 120 \mathrm{mmHg}$, DBP $\leq 80 \mathrm{mmHg}$, no current smoking, no diabetes and no prior CVD [10, 22].

According to the 2013 ACC/AHA guidelines, statin therapy should be recommended for patients if they meet the following criteria: age $\geq 21$ years with LDLc $\geq 4.9 \mathrm{mmol} / \mathrm{l}$, age $40-75$ years with diabetes and LDL-c $1.8-4.9 \mathrm{mmol} / \mathrm{l}$, or age $40-75$ years with 10 -year ASCVD risk score of $7.5 \%$ or higher [23]. We determined the number of patients in our cohort who should be on statin therapy according to these guidelines.

\section{Ethics}

Written informed consent was obtained from all individual participants prior to enrollment in the parent trials. The two studies were reviewed and approved by the institutional review board of the Peking Union Medical College Hospital prior to initiation. All procedures performed were carried out in accordance with the ethical standards laid down in the 1964 Declaration of Helsinki and its later amendments.

\section{Statistical analysis}

All statistical analyses were performed using the SPSS 19.0 statistical software package (IBM Corporation, Armonk, New York, USA). Descriptive data were tabulated and reported using simple means, standard deviations, medians, interquartile ranges, and frequencies. Stratified analyses based upon gender and age strata (20-29, 30-39, 40-49, 50-59, $\geq 60$ years) were assessed using the Student's $t$-test for parametric continuous variables, Wilcoxon Rank Sum test for non-parametric continuous variables, and the Chi-squared test for categorical variables. Univariate and multivariable logistic regression [24] were performed to assess the relationship between the dependent variables of interest (FRS $\geq 10 \%$, $\mathrm{D}: \mathrm{A}: \mathrm{D}$ risk score $\geq 10 \%$, and ASCVD risk score $\geq 10 \%$ ) and the independent variables of interest [age, sex, current smoking, BMI, hypertension, diabetes, dyslipidemia, transmission route, baseline CD4+ cell count (categorized as $<200,200-349$, and $>350$ cells $/ \mathrm{mm}^{3}$ ), and baseline viral load]. Variables with $p<0.2$ in the univariate analyses were included in multivariable regression. All tests performed were two-tailed, with $p<0.05$ considered to be statistically significant.

\section{Results}

\section{Sociodemographic and clinical characteristics}

Table 1 summarizes the characteristics of the 973 Chinese adult ART-naïve patients whose data were included in the study. The patient population was primarily male (722/ $973,74.2 \%)$. The mean age was $36.0 \pm 10.2$ years, ranging from 18 to 65 years. Mean CD4+ cell count was $229 \pm 123$ cells $/ \mathrm{mm}^{3}$, and viral load was $4.69 \pm 0.70 \log 10$ copies $/ \mathrm{ml}$. Compared to our sample, the overall population of 1607 patients from the two parent trials was older (38.0 \pm 11.0 years, $p<0.001)$ and had a somewhat lower proportion of male participants [1132 (70.4\%), $p=0.039$ ], however did not differ significantly based upon other clinical or CVD risk characteristics.

The most common CVD risk factors in this cohort were dyslipidemia (51.7\%) and current smoking (23.7\%). The prevalence of hypertension, diabetes and obesity were $8.4 \%, 4.6 \%$ and $1.0 \%$, respectively. The majority of patients $(67.7 \%)$ had at least one CVD risk factor (current smoking, obesity, diabetes, hypertension or dyslipidemia), and $19.9 \%$ had two or more risk factors. Based upon the NCEP-ATP III guidelines, $10.7 \%$ of individuals in our cohort met criteria for elevated TC levels, 29.0\% had elevated TG levels, 30.7\% had low HDL-c levels, and 1.7\% had elevated LDL-c levels (Table 1).

Compared with female patients (Table 1), male patients were younger, had a higher prevalence of smoking and dyslipidemia, and higher viral loads. Among those meeting criteria for dyslipidemia, women were more likely than men to have elevated TC and LDL-c thresholds, whereas men were more likely than women to have low HDL-c levels $(p<0.05)$. Finally, a greater proportion of male patients had at least one CVD risk factor (72.7\%) compared with their female counterparts (53.4\%).

\section{Cardiovascular disease risk}

No patients had a known history of CVD at the time of enrollment. Table 2 shows the 10 -year estimated CVD risk using the Framingham and D:A:D risk equations. Among 965 patients $\geq 20$ years of age, 39 patients (4.0\%) had a FRS between 10 and $20 \%$, and five individuals $(0.5 \%)$ had a FRS $\geq 20 \%$. Among 973 patients, $20(2.1 \%)$ patients had a D:A:D risk score between 10 and 20\%, and 
Table 1 Demographic and clinical characteristics of study participants, overall and by sex

\begin{tabular}{|c|c|c|c|c|}
\hline Characteristic & All $(n=973)$ & Male $(n=722)$ & Female $(n=251)$ & $p$-value \\
\hline Age years & $36.0 \pm 10.2$ & $35.1 \pm 10.1$ & $38.7 \pm 10.1$ & $<0.001$ \\
\hline Route of transmission & & & & $<0.001^{\mathrm{b}}$ \\
\hline MSM & $382(39.3 \%)$ & $382(52.9 \%)$ & 0 & \\
\hline Heterosexual & $466(47.9 \%)$ & $248(34.3 \%)$ & $218(86.9 \%)$ & \\
\hline Blood-borne & $51(5.2 \%)$ & $32(4.4 \%)$ & $19(7.6 \%)$ & \\
\hline Other/unknown & $74(7.6 \%)$ & $60(8.3 \%)$ & $14(5.6 \%)$ & \\
\hline Years since HIV diagnosis & $1.0 \pm 1.6$ & $0.8 \pm 1.3$ & $1.6 \pm 2.1$ & 0.01 \\
\hline CD4+ count cells $/ \mathrm{mm}^{3}(N=969)$ & $229 \pm 123$ & $227 \pm 124$ & $233 \pm 121$ & 0.52 \\
\hline HIV RNA log10 copies/ml $(N=710)$ & $4.69 \pm 0.70$ & $4.74 \pm 0.67$ & $4.56 \pm 0.77$ & $<0.01$ \\
\hline Current smoking & $231(23.7 \%)$ & $223(30.9 \%)$ & $8(3.2 \%)$ & $<0.001$ \\
\hline $\mathrm{BMI} \mathrm{kg} / \mathrm{m}^{2}$ & $21.6 \pm 2.9$ & $21.5 \pm 2.7$ & $21.6 \pm 3.3$ & 0.82 \\
\hline Overweight & $94(9.7 \%)$ & $63(8.7 \%)$ & $31(12.4 \%)$ & 0.09 \\
\hline Obesity & $10(1.0 \%)$ & $5(0.7 \%)$ & $5(2.0 \%)$ & 0.08 \\
\hline Systolic blood pressure mmHg & $115 \pm 13$ & $116 \pm 12$ & $113 \pm 14$ & $<0.01$ \\
\hline Diastolic blood pressure mmHg & $75 \pm 9$ & $75 \pm 9$ & $75 \pm 10$ & 0.79 \\
\hline Hypertension & $82(8.4 \%)$ & $56(7.8 \%)$ & $26(10.4 \%)$ & 0.20 \\
\hline Blood glucose mmol/l & $5.3 \pm 1.0$ & $5.3 \pm 1.0$ & $5.3 \pm 1.1$ & 0.77 \\
\hline Diabetes & $45(4.6 \%)$ & $33(4.6 \%)$ & $12(4.8 \%)$ & 0.89 \\
\hline TC mmol/l & $4.1 \pm 1.0$ & $4.0 \pm 0.9$ & $4.2 \pm 1.1$ & $<0.01$ \\
\hline $\mathrm{TG} \mathrm{mmol} / \mathrm{l}$ & $1.5 \pm 1.1$ & $1.5 \pm 1.1$ & $1.5 \pm 1.0$ & 0.21 \\
\hline HDL-c mmol/l & $1.2 \pm 0.4$ & $1.2 \pm 0.4$ & $1.3 \pm 0.4$ & $<0.001$ \\
\hline LDL-c mmol/l & $2.3 \pm 0.8$ & $2.3 \pm 0.8$ & $2.3 \pm 0.9$ & 0.95 \\
\hline $\mathrm{TC}>5.2 \mathrm{mmol} / \mathrm{l}$ & $104(10.7 \%)$ & 66 (9.1\%) & $38(15.1 \%)$ & $<0.01$ \\
\hline $\mathrm{TG}>1.7 \mathrm{mmol} / \mathrm{l}$ & $282(29.0 \%)$ & $215(29.8 \%)$ & $67(26.7 \%)$ & 0.35 \\
\hline $\mathrm{HDL}-\mathrm{c}<1.0 \mathrm{mmol} / \mathrm{l}$ & $299(30.7 \%)$ & $249(34.5 \%)$ & $50(19.9 \%)$ & $<0.001$ \\
\hline $\mathrm{LDL-C}>4.1 \mathrm{mmol} / \mathrm{l}$ & $17(1.7 \%)$ & $9(1.2 \%)$ & $8(3.2 \%)$ & 0.04 \\
\hline Dyslipidemia & $503(51.7 \%)$ & $388(53.7 \%)$ & $115(45.8 \%)$ & 0.03 \\
\hline \multicolumn{5}{|l|}{ Number of CVD risk factors ${ }^{a}$} \\
\hline Only one & $465(47.8 \%)$ & $361(50.0 \%)$ & $104(41.4 \%)$ & 0.019 \\
\hline Two or more & $194(19.9 \%)$ & $164(22.7 \%)$ & $30(12.0 \%)$ & $<0.001$ \\
\hline
\end{tabular}

Values are $n(\%)$ or mean \pm standard deviation

MSM, men who have sex with men; BMI, body mass index; TC, total cholesterol; TG, triglycerides; HDL-c, high-density lipoprotein cholesterol; LDL-c, low-density lipoprotein cholesterol; CVD, cardiovascular disease; NA, not applicable

${ }^{a}$ Composite of current smoking, obesity $\left(B M I \geq 30.0 \mathrm{~kg} / \mathrm{m}^{2}\right)$, hypertension, diabetes, or dyslipidemia

${ }^{\mathrm{b}}$ Not including MSM category

$12(1.2 \%)$ had a D:A:D risk score $\geq 20 \%$. The proportion of patients with $>10 \%$ estimated10-year risk of CVD were $4.5 \%$ using the FRS and $3.3 \%$ using D:A:D $(p=0.15)$. All patients with FRS $\geq 10 \%$ were male. As shown in Fig. 1, all patients with FRS or D:A:D risk scores $\geq 10 \%$ were older than 40 years of age, and there were no differences in the proportion of individuals with 10 -year estimated CVD risk $\geq 10 \%$ across different age strata between the two risk scores.

In the multivariable logistic regression analysis (Table 3), while older age and smoking were positively associated with FRS $\geq 10 \%$ and ASCVD $\geq 10 \%$, HIV-specific parameters such as route of transmission, baseline viral load and $\mathrm{CD} 4+\mathrm{T}$ cell count were not independent risk factors for 10-year risk of CHD using any of the CVD risk prediction models. None of the predictors were found to be significantly associated with D:A:D Risk Score in the multivariable model (data not shown).

The ASCVD Risk Score was calculated in 247 of 307 patients who were $\geq 40$ years of age. In the multivariable logistic regression analysis (Table 3), older age and smoking were positively associated with ASCVD $\geq 10 \%$. As shown in Fig. 2, the proportions of patients with $\geq 10 \%$ estimated 10 -year risk of CVD 
Table 2 Estimated 10-year cardiovascular risk among Chinese antiretroviral-naïve HIV-infected patients

\begin{tabular}{llll}
\hline Characteristics & All $n / N(\%)$ & Male $n / N(\%)$ & Female $n / N(\%)$ \\
\hline FRS & & & $p$-value \\
$\quad<10 \%$ & $921 / 965(95.5 \%)$ & $671 / 715(93.8 \%)$ & $250 / 250(100 \%)$ \\
$10-20 \%$ & $39 / 965(4.0 \%)$ & $39 / 715(5.5 \%)$ & $0 / 250(0 \%)$ \\
$\quad>20 \%$ & $5 / 965(0.5 \%)$ & $5 / 715(0.7 \%)$ & $0 / 250(0 \%)$ \\
D:A:D Risk Score & & & NA \\
$\quad<10 \%$ & $941 / 973(96.7 \%)$ & $692 / 722(95.8 \%)$ & $249 / 251(99.2 \%)$ \\
$\quad 10-20 \%$ & $20 / 973(2.1 \%)$ & $18 / 722(2.5 \%)$ & $2 / 251(0.8 \%)$ \\
$\quad>20 \%$ & $12 / 973(1.2 \%)$ & $12 / 722(1.7 \%)$ & $0 / 251(0 \%)$ \\
Favorable Cardiovascular Risk Profile & $364 / 973(37.4 \%)$ & $257 / 722(35.6 \%)$ & $107 / 251(42.6 \%)$
\end{tabular}

NA, not applicable; FRS, the Framingham Risk Score; (D:A:D) Risk Score, Data Collection on Adverse Events of Anti-HIV Drugs (D:A:D) Risk Scores; Favorable cardiovascular risk profile was defined on the basis of a number of modifiable risk factors as follows: total cholesterol $<5.17 \mathrm{mmol} / \mathrm{l}$, systolic blood pressure $\leq 120 \mathrm{mmHg}$, diastolic blood pressure $\leq 80 \mathrm{mmHg}$, no current smoking, no diabetes and no prior CVD

was $16.6 \%$ using the FRS, $12.1 \%$ using D:A:D risk estimators and $10.5 \%$ using the ASCVD Risk Score. In general, the D:A:D and ASCVD Risk Scores were not significantly different, however FRS Risk Scores were significantly higher than estimates derived from the other two prediction models $(p=0.01)$.

By contrast, $37.4 \%$ of patients had a favorable CVD risk profile, with women being more likely to have a favorable risk profile compared with men $(p=0.047)$ (Table 2).

\section{Treatment patterns for cardiovascular risk factors}

Among the 973 patients in this cohort, 47(4.8\%) patients (2 patients with LDL-c $\geq 4.91 \mathrm{mmol} / \mathrm{l} ; 15$ patients $>40$ years, with diabetes and LDL-c 1.81-4.91 mmol/l; 30 patients $>40$ years with ASCVD Risk Score $\geq 7.5 \%$ ) met criteria for initiation of statin therapy according to the 2013 ACC/AHA guidelines. However, no patients in our study were currently being treated with statins.

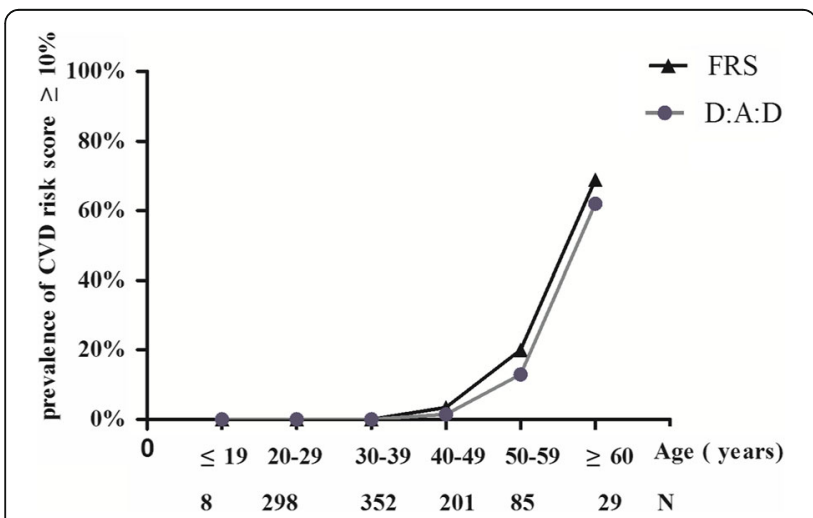

Fig. 1 Prevalence of individuals with 10-year risk of CHD $\geq 10 \%$ according to the FRS and D:A:D Risk Score. FRS: the Framingham risk score; D:A:D: Data Collection on Adverse Events of Anti-HIV Drugs Risk Score
Furthermore, only 5.6\% (10/179) of patients with hypertension were currently receiving antihypertensive therapy, and only $4.6 \%(4 / 87)$ of patients meeting criteria for diabetes were currently receiving pharmacologic treatment for diabetes.

\section{Discussion}

This is the first multicenter study in China, and one of the largest in Asia, to comprehensively assess CVD risk among adult ART-naïve HIV-infected patients using composite CVD risk scores, and to assess gaps in treatment of key modifiable CVD risk factors. While overall 10-year CVD risk based upon the FRS and D:A:D risk score was low, likely due to the young age of this cohort and comparatively low BMI, we nonetheless found that over $65 \%$ of individuals had at least one CVD risk factor, and that few patients with hypertension, diabetes or dyslipidemia were prescribed treatment.

Mortality from CVD has risen dramatically in China over the past two decades and is now the leading cause of death among Chinese men and women [13]. This is due to increasing prevalence of CVD risk factors (e.g. diabetes, hypertension, and dyslipidemia) driven by aging, dietary change, physical inactivity, increasing BMI, and strikingly high rates of smoking and second hand smoke exposure. Therefore, understanding baseline CVD risk among individuals with HIV in China, and ensuring early intervention for individuals with CVD risk factors is highly important.

For the purposes of this study, we were not able to recruit a comparator group of HIV-negative individuals with similar demographic and HIV risk characteristics. However, data from the general Chinese population provide us with a basis for drawing some comparisons. The China Noncommunicable Disease Surveillance Group carried out a study among a nationally representative sample of over 90,000 Chinese adults over 20 years 
Table 3 Logistic regression analysis of 10-year CVD risk $\geq 10 \%$, by FRS or ASCVD risk score

\begin{tabular}{|c|c|c|c|c|}
\hline \multirow[t]{2}{*}{ Variable } & \multicolumn{2}{|l|}{ FRS $\geq 10 \%$} & \multicolumn{2}{|l|}{$A S C V D \geq 10 \%$} \\
\hline & $\begin{array}{l}\text { Crude OR } \\
(95 \% \mathrm{Cl})\end{array}$ & $\begin{array}{l}\text { Adjusted } \mathrm{OR}^{\mathrm{a}} \\
(95 \% \mathrm{Cl})\end{array}$ & $\begin{array}{l}\text { Crude OR } \\
(95 \% \mathrm{Cl})\end{array}$ & $\begin{array}{l}\text { Adjusted } \mathrm{OR}^{\mathrm{a}} \\
(95 \% \mathrm{Cl})\end{array}$ \\
\hline Age years & $1.28(1.21,1.35)^{\#}$ & $1.42(1.27,1.59){ }^{\#}$ & $1.35(1.23,1.49){ }^{\#}$ & $1.80(1.28,2.53)^{\$}$ \\
\hline Female & NA & - & NA & - \\
\hline Current smoking & $6.88(3.62,13.09) \#$ & $61.70(11.35,335.57) \#$ & $11.63(4.43,30.56) \#$ & $334.16(8.25,13,531.31)^{\$}$ \\
\hline BMl & $1.07(0.97,1.18)$ & - & $0.93(0.791 .09)$ & - \\
\hline Hypertension & $3.99(1.94,8.23)^{\#}$ & $2.26(0.54,9.39)$ & $2.16(0.84,5.56)^{\dagger}$ & $2.82(0.32,25.29)$ \\
\hline Diabetes & $7.45(3.41,16.27){ }^{\#}$ & $1.33(0.20,8.85)$ & $10.79(4.18,27.84) \#$ & $10.58(0.60,185.97)$ \\
\hline Dyslipidemia & $2.33(1.20,4.51)^{+}$ & $2.44(0.67,8.85)$ & $1.36(0.58,3.18)$ & - \\
\hline \multicolumn{5}{|l|}{ Route of transmission } \\
\hline MSM & Reference & Reference & Reference & \\
\hline Heterosexual & $2.43(1.17,5.05)^{+}$ & $0.24(0.04,1.31)$ & $1.31(0.46,3.71)$ & - \\
\hline Blood & $1.49(0.32,7.00)$ & NA & $0.75(0.08,7.03)$ & - \\
\hline Other/unknown & $1.54(0.41,5.75)$ & $0.17(0.02,1.96)$ & $0.85(0.154 .73)$ & - \\
\hline Years since HIV diagnosis & $0.90(0.71,1.14)$ & - & $0.53(0.27,1.05)$ & - \\
\hline \multicolumn{5}{|l|}{ CD4+ count cells/ mm ${ }^{3}$} \\
\hline$<200$ & $1.71(0.63,4.64)$ & - & $1.17(0.31,4.47)$ & - \\
\hline $200-349$ & $1.50(0.55,4.08)$ & - & $1.47(0.39,5.55)$ & - \\
\hline$>350$ & Reference & Reference & Reference & Reference \\
\hline Viral load log10 copies/ml & $1.67(1.02,2.73)^{+}$ & $0.80(0.38,1.71)$ & $1.84(0.97,3.49)^{\dagger}$ & $1.93(0.54,6.86)$ \\
\hline
\end{tabular}

*Factors with $p<0.2$ in the univariate analyses were included in multivariable regression. OR: Odds ratio; Cl: Confidence interval; BMI: body mass index; MSM: Men who have sex with men; FRS, the Framingham Risk Score; ASCVD, Atherosclerotic Cardiovascular Disease Risk Score; NA: not applicable ${ }^{+} p<0.2,{ }^{+} p<0.05,{ }^{\$} p<0.01,{ }^{*} p<0.001$

of age, and found that the prevalence of smoking, overweight and obesity, hypertension, dyslipidemia, and diabetes were $58.16,36.67,30.09,67.43$, and $10.61 \%$, respectively, among men; and 3.44, 29.77, 24.79, 63.98, and $8.73 \%$, respectively, among women. [11]. Another recent study evaluating cardiovascular risk factors among a nationally representative sample of 23,010

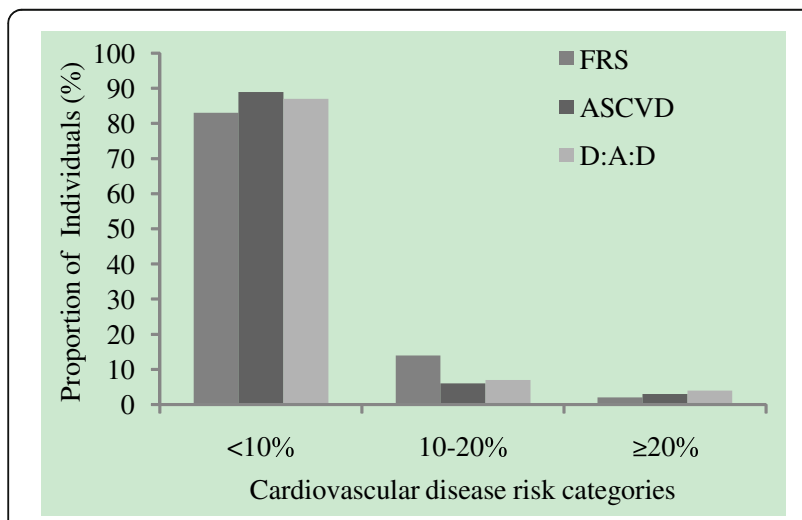

Fig. 2 Cardiovascular disease (CVD) risk categories, by risk score. Ten-year CVD risk is depicted in three categories: $<10 \%, 10-20 \%$ and $>20 \%$, for each of the three CVD risk prediction models. ASCVD: atherosclerotic cardiovascular disease risk score; FRS: the Framingham risk score; D:A:D: Data Collection on Adverse Events of Anti-HIV Drugs Risk Score
Chinese adults aged 18 years and older found the prevalence of smoking, overweight and obesity, hypertension, dyslipidemia, and diabetes were 43.2, 37.2, 27.4, 55.2, and $5.0 \%$ respectively among men; and $2.9,27.6,21.6$, 44.3 , and $3.7 \%$ respectively among women [12]. Of note, the former study, which was published in 2012, used slightly different criteria to define these CVD risk factors, but the latter study, published in 2016, utilized the same definitions for CVD risk factors applied in our analysis. Among the treatment-naïve adults with HIV in our study, prevalence of hypertension and overweight/obesity were notably lower that that reported in the general population. This finding likely reflects the catabolic status among our participants in the setting of advanced HIV disease status, as evidenced by the low mean CD4+ T cell count of $229 \pm 123$ cells $/ \mathrm{mm}^{3}$. However, interestingly, rates of dyslipidemia and diabetes were similar compared to the general population despite the lower mean BMI of this population, perhaps signifying altered lipid and glucose metabolism in the setting of untreated HIV infection. The prevalence of current smoking among patients in our study was lower than found in the two general population studies [11,12]. While smoking data was systematically collected for all participants, we cannot exclude the possibility of underreporting of this risk factor. 
In terms of other cardiovascular endpoints, our group has previously published echocardiographic data collected in a subgroup of participants from the China AIDS Clinical Trial 0810 [25]. At baseline, prior to ART initiation, the prevalence of echocardiographic abnormalities, including left ventricular systolic dysfunction and diastolic dysfunction, was significantly higher among 325 ARTnaive persons living with HIV compared with 97 age- and sex-matched healthy controls, underscoring again the risk for CVD among individuals with HIV.

The Strategic Timing of Anti-Retroviral Treatment (START) trial, which recruited ART-naïve patients from six continents, found that the proportion of individuals having at least one CVD risk factor varied widely by geographic region (North America:70\%; Europe/Australia/ Israel: 65.1\%; South America:49.4\%; Asia: 37\%; and Africa: 55.7\%) [10]. Compared to the Asian cohort in the START trial, composed of 154 individuals with HIV from Thailand, participants in our cohort were older [34(2842) v. 30(24-37) years] and had a notably lower median CD4+ T cell count [235(141-314) v. 604(561-677) cells $/ \mathrm{mm}^{3}$ ]. While prevalence of hypertension was similar, patients in our cohort had a higher prevalence of diabetes (4.6\% v. $1.3 \%)$, dyslipidemia $(51.7 \%$ v. $13.0 \%)$ and smoking $(23.7 \%$ v. $16.9 \%)$, but lower prevalence of obesity (1\% v. $3.2 \%)$.

It is important to note, however, that in the START trial, dyslipidemia was defined by LDL-c $\geq 4.1 \mathrm{mmol} / \mathrm{l}$ or use of cholesterol-lowering drugs, which differs from the definition used in our study. Adopting the definition used by the START trial, the prevalence of dyslipidemia in our population decreased to $1.7 \%$, and the prevalence of at least one CVD risk factor decreased from $67.8 \%$ to $35 \%$. However, in our cohort, the majority of dyslipidemia was attributable to high TG and low HDL, which is consistent with previous published studies in low- and middle-income countries, including a recent study by Shen et al. focused on prevalence of dyslipidemia among Chinese ART-naïve patients in China [26-30]. This, combined with the significant rate of under-treatment of dyslipidemia in our population, led us to utilize the more comprehensive definition put forth by the NCEP-ATP III Guidelines.

In addition to CVD risk factor prevalence data, our study provides novel information regarding 10-year CVD risk among patients in our cohort. We chose to use the FRS because it has been used widely both internationally and in China to predict CVD risk among HIV-negative patients [31], and FRS has good predictive accuracy for subclinical carotid atherosclerosis [32]. The D:A:D risk equations were specifically constructed for use in HIVinfected populations [4]. Given the younger age of populations with HIV in China (68.4\% of patients in our cohort were less than 40 years), the FRS and D:A:D risk equations are more widely applicable compared with tools such as the ACC/AHA ASCVD Risk Score, which applies to individuals $\geq 40$ years of age. The prevalence of patients with 10 -year CVD risk $\geq 10 \%$ in our cohort was $4.5 \%$ based upon the FRS, which is on par with that reported previously among other studies among Asian (6.5\%) and African HIV-positive patients (3.6\%) [10, 33] and lower compared with rates observed among individuals with HIV in Western countries (19.6-21.1\%) [10]. The lower CVD risk in Asia and Africa in the START trial may reflect younger age and lower hypertension and obesity rates for the former, and higher proportion of women and low smoking and dyslipidemia rates in the latter. Consistent with these findings, patients in our study had lower rates of hypertension and obesity, but also more advanced HIV disease contributing to lower overall risk for CVD risk.

Among patients $\geq 40$ years of age, lower overall CVD risk was attributed to the ART-naïve HIV-infected patients using the ASCVD model and the D:A:D risk score compared with the FRS. Prior studies have also suggested that the FRS overestimates CVD risk among individuals with HIV [34], however other studies have raised concerns about underestimation of CVD risk among individuals with HIV using the FRS as well [11]. In our cohort, the FRS and ASCVD Risk Scores both identified age and smoking as correlates of 10-year CVD risk $\geq 10 \%$, which is not surprising given they are factored into the risk models themselves. However after adjusting for traditional risk factors no associations were observed between HIV-related factors and 10-year CVD risk $\geq 10 \%$, irrespective of the risk algorithm used, similar to previous findings [35]. The D:A:D CVD Risk Score was developed and validated in a cohort of patients with HIV for the prediction of 5-year CVD risk, however the cohort used to develop the tool was largely European and American, and all patients were on ART. While it has been applied previously to study 10-year CVD risk in both treated and treatment-naïve patients with HIV [9, 10], the direct applicability of this algorithm to a relatively young treatment-naïve population in a low- and middle-income setting still requires formal validation, and findings should be interpreted with this caveat in mind.

Finally, prior studies from Western countries have highlighted the problem of underdiagnosis and undertreatment of CVD risk factors among ART-naïve HIV-infected patients [33]. One study from Italy demonstrated approximately $50 \%$ patients with HIV meeting criteria for statin therapy were not being treated [36]. Another study from France found HIV-infected patients treated with statins after acute coronary syndrome had less of an improvement in lipid profiles when compared with HIV-negative controls, in the setting of less potent statins and potential drug-drug 
interactions with antiretroviral drugs [37]. De Socio et al. found that one- to two-thirds of hypertensive HIV-infected patients in their study were unaware of their condition, and were not on antihypertensive therapy [38]. Furthermore, Zanni et al. found that compared with 2004 ATP III guidelines, under the 2013 ACC/AHA guidelines a higher percentage of patients met criteria for statin therapy, however, when patients were evaluated with contrast-enhanced coronary computed tomography angiography, significant discordance was observed between those initiated on statins and those found to have high risk morphology (HRM) coronary plaques. In fact the authors found that $74 \%$ of those with subclinical HRM coronary plaque would not be recommended statin therapy based upon the 2013 ACC/AHA guidelines alone [39]. Therefore, in addition to addressing underdiagnosis and undertreatment of CVD risk factors among individuals with HIV, future studies are also needed to evaluate the appropriateness of applying treatment guidelines developed for the general population to this population.

Few studies, however, have addressed this issue in resource-limited settings. In our cohort, we found significant under-treatment of dyslipidemia, hypertension and diabetes. Approximately $68 \%$ of individuals in our study had at least one risk factor for CVD. National clinical guidelines for HIV/AIDS care dictate that blood pressure is measured, and fasting lipid profile and glucose are checked prior to initiation of ART. However, care providers may be less well versed in the nuances of actual management of these primary care issues, particularly thresholds for initiating lipid lowering therapy. Furthermore, smoking cessation should be a cornerstone of primary CVD prevention, but is inadequately addressed in most HIV care settings in China. Further research is necessary to identify the prevention strategies that are most feasible and effective for reducing CVD risk within our population, and should combine both life-style modification strategies with pharmacologic management of hypertension, diabetes, and dyslipidemia $[5,14]$. Although the currently existing CVD risk scores may not have been designed with our population in mind, they nevertheless provide a starting point for care providers to evaluate key CVD risk factors and identify individuals at highest-risk who may need close evaluation and management.

Our study has several limitations. First, our data are cross-sectional and therefore do not provide information regarding longitudinal change in CVD risk over time after initiation of ART, or regarding CVD events. Second, patients with CD4+ cell count $\geq 500$ cells $/ \mathrm{mm}^{3}$ were not enrolled in our study, which may explain why we did not see an association between CD4+ cell count and
CVD risk. However, lack of correlation between CD4+ cell count and CVD risk has also been reported by Sabin et al. [40]. Third, the original studies from which these data are drawn were not designed with CVD risk assessment as their primary endpoints, therefore our analysis focuses on the subset of 973 patients who had complete CVD risk factor data available, which may influence the generalizability of our results. However, there is no known systematic bias influencing collection of risk factor data, and risk factor prevalence in the overall group was similar to that of the subset analyzed. Finally, in our study we did not have data on actual CVD clinical outcomes such as stroke or ischemic heart disease which would have enabled us to assess the ability of the FRS and ASCVD Risk Score to predict CVD in our cohort.

\section{Conclusions}

In conclusion, although baseline predicted 10-year risk of CVD was low in this relatively young population, we found that CVD risk factors were common and significantly under-treated. More research is needed to understand the unique biology and epidemiology of HIV-associated CVD in Asia, to identify the optimal tools to assess CVD risk among individuals with HIV in Asia, and to assess the impact of prolonged infection and exposure to ART on CVD outcomes in this population. Finally, future interventions should focus on training HIV providers in China to appropriately recognize and manage CVD risk factors during routine clinical assessments.

\section{Abbreviations}

ACC/AHA: American College of Cardiology/American Heart Association; ART: Antiretroviral therapy; ASCVD: Atherosclerotic Cardiovascular Disease; BMI: Body mass index; CVD: Cardiovascular disease; D:A:D: Data Collection on Adverse Events of Anti-HIV Drugs; DBP: Diastolic blood pressure;

FRS: Framingham Risk Score; HDL-c: High-density lipoprotein cholesterol; HRM: High risk morphology; LDL-c: Low-density lipoprotein cholesterol; NCEP-ATP: National Cholesterol Education Program, Adult Treatment Panel; SBP: Systolic blood pressure; TC: Total cholesterol; TG: Triglycerides

\section{Acknowledgements}

We thank the patients and their families for their participation and support during this study. The following clinical institutions or hospitals participated in this study: The Infectious Disease Hospital of Henan Province; Shanghai Public Health Clinical Center Affiliated to Fudan University; Fuzhou Infectious Diseases Hospital Affiliated to Fujian Medical University; Guangzhou No. 8 People's Hospital; Shenzhen Third People's Hospital; Beijing You'an Hospital, Capital Medical University; Beijing Ditan Hospital, Capital Medical University; Yunnan AIDS Care Center; The First People's Hospital of Honghe State; Tangdu Hospital Affiliated to the Fourth Military Medical University, Xi'an; Kunming Third People's Hospital; Chengdu Infectious Diseases Hospital; Longtan Hospital of Guangxi Zhuang Autonomous Region; The First Hospital of Changsha; The First Affiliated Hospital of China Medical University. Special thanks to Michael T. Yin (Columbia University Medical Center, New York, New York) for his valuable input during the preparation of this manuscript.

\section{Funding}

This study was funded by the National Key Technologies R\&D Program for the 12th Five-year Plan (2012ZX10001003-001). 


\section{Availability of data and materials}

The datasets used and/or analysed in the current study are available from the corresponding author on reasonable request. Special administrative permissions, other than permission from the corresponding author, were not required in order to use the data described in this manuscript.

\section{Authors' contributions}

$F G, E H, W L$ and $T L$ participated in the conception and design of the study. FG and EH performed the data analysis and drafted the manuscript. YH, JX, $Y L$ and XS performed the original data collection (including patient recruitment, laboratory analyses and clinical data collection) and provided direct input to development of the methodology section for this manuscript. TL supervised this study. All authors reviewed and revised the draft, and approved the final version of the manuscript.

\section{Competing interests}

The authors declare that they have no competing interest.

\section{Consent for publication}

\section{Not applicable.}

\section{Ethics approval and consent to participate}

The two studies were reviewed and approved by the institutional review board of the Peking Union Medical College Hospital prior to initiation. Written informed consent was obtained from all individual participants prior to enrollment in the parent trials. All procedures performed were carried out in accordance with the ethical standards laid down in the 1964 Declaration of Helsinki and its later amendments. Based upon the policy of the institutional review board of the Peking Union Medical College Hospital, no formal additional ethics approval was required for the current analysis as no additional data was collected and all data analyzed were de-identified.

\section{Publisher's Note}

Springer Nature remains neutral with regard to jurisdictional claims in published maps and institutional affiliations.

\section{Author details}

'Department of Infectious Diseases, Peking Union Medical College Hospital, Chinese Academy of Medical Sciences, Beijing, China. ${ }^{2}$ Department of Internal Medicine, Yale School of Medicine, New Haven, CT, USA.

Received: 15 January 2017 Accepted: 28 March 2017

Published online: 20 April 2017

\section{References}

1. Freiberg MS, Chang CC, Kuller LH, Skanderson M, Lowy E, Kraemer KL, et al. HIV infection and the risk of acute myocardial infarction. JAMA Intern Med. 2013;173(8):614-22.

2. Data Collection on Adverse Events of Anti-HIV drugs (D:A:D) Study Group, Smith C, Sabin CA, Lundgren JD, Thiebaut R, Weber R, et al. Factors associated with specific causes of death amongst HIV-positive individuals in the D:a:D study. AIDS. 2010;24(10):1537-48.

3. Grinspoon SK, Grunfeld C, Kotler DP, Currier JS, Lundgren JD, Dube MP, et al. State of the science conference: initiative to decrease cardiovascular risk and increase quality of care for patients living with HIV/AIDS: executive summary. Circulation. 2008;118(2):198-210.

4. Friis-Moller $\mathrm{N}$, Thiebaut R, Reiss $\mathrm{P}$, Weber R, Monforte AD, De Wit S, et al. Predicting the risk of cardiovascular disease in HIV-infected patients: the data collection on adverse effects of anti-HIV drugs study. Eur J Cardiovasc Prev Rehabil. 2010;17(5):491-501.

5. Martin-Iguacel R, Llibre JM, Friis-Moller N. Risk of cardiovascular disease in an aging HIV population: where are we now? Curr HIV/AIDS Rep. 2015;12(4):375-87.

6. Lang S, Mary-Krause M, Cotte L, Gilquin J, Partisani M, Simon A, et al. Increased risk of myocardial infarction in HIV-infected patients in France, relative to the general population. AIDS. 2010;24(8):1228-30.

7. Kaplan RC, Kingsley LA, Sharrett AR, Li X, Lazar J, Tien PC, et al. Ten-year predicted coronary heart disease risk in HIV-infected men and women. Clin Infect Dis. 2007:45(8):1074-81.

8. Wilson PW, D'Agostino RB, Levy D, Belanger AM, Silbershatz H, Kannel WB. Prediction of coronary heart disease using risk factor categories. Circulation. 1998;97(18):1837-47.
9. Edwards-Jackson N, Kerr S, Tieu H, Ananworanich J, Hammer S, Ruxrungtham K, et al. Cardiovascular risk assessment in persons with HIV infection in the developing world: comparing three risk equations in a cohort of HIV-infected Thais. HIV Med. 2011;12(8):510-5.

10. Soliman EZ, Sharma S, Arasteh K, Wohl D, Achhra A, Tambussi G, et al. Baseline cardiovascular risk in the INSIGHT strategic timing of AntiRetroviral treatment (START) trial. HIV Med. 2015;16(Suppl 1):46-54.

11. Yang ZJ, Liu J, Ge JP, Chen L, Zhao ZG, Yang WY, et al. Prevalence of cardiovascular disease risk factor in the Chinese population: the 2007-2008 China National Diabetes and metabolic disorders study. Eur Heart J. 2012;33(2):213-20.

12. Wu J, Cheng X, Qiu L, Xu T, Zhu G, Han J, et al. Prevalence and clustering of major cardiovascular risk factors in China: a recent cross-sectional survey. Medicine. 2016;95(10):e2712.

13. Yang G, Wang Y, Zeng Y, Gao GF, Liang X, Zhou M, et al. Rapid health transition in China, 1990-2010: findings from the global burden of disease study 2010. Lancet. 2013;381(9882):1987-2015.

14. Li T, Guo F, Li Y, Zhang C, Han Y, Lye W, et al. An antiretroviral regimen containing 6 months of stavudine followed by long-term zidovudine for first-line HIV therapy is optimal in resource-limited settings: a prospective, multicenter study in China. Chin Med J. 2014;127(1):59-65.

15. Fan HW, Guo FP, Li YJ, Li N, Li TS. Prevalence of thrombocytopenia among Chinese adult antiretroviral-naive HIV-positive patients. Chin Med J. 2015; 128(4):459-64.

16. Clinical Guidelines on the Identification, Evaluation, and Treatment of Overweight and Obesity in Adults-The Evidence Report. National Institutes of Health. Obesity research. 1998; 6 Suppl 2:51S-209S.

17. Pickering $T G$, Hall JE, Appel LJ, Falkner BE, Graves J, Hill MN, et al. Recommendations for blood pressure measurement in humans and experimental animals: part 1: blood pressure measurement in humans: a statement for professionals from the Subcommittee of Professional and Public Education of the American Heart Association Council on high blood pressure research. Hypertension. 2005;45(1):142-61.

18. National Cholesterol Education Program Expert Panel on Detection E, Treatment of High Blood Cholesterol in A. Third report of the National Cholesterol Education Program (NCEP) expert Panel on Detection, evaluation, and treatment of high blood cholesterol in adults (adult treatment Panel III) final report. Circulation. 2002;106(25):3143-421.

19. Bergersen BM, Sandvik L, Bruun JN, Tonstad S. Elevated Framingham risk score in HIV-positive patients on highly active antiretroviral therapy: results from a Norwegian study of 721 subjects. Eur J Clin Microbiol Infect Dis. 2004;23(8):625-30.

20. Krikke M, Hoogeveen RC, Hoepelman Al, Visseren FL, Arends JE. Cardiovascular risk prediction in HIV-infected patients: comparing the Framingham, atherosclerotic cardiovascular disease risk score (ASCVD), systematic coronary risk evaluation for the Netherlands (SCORE-NL) and data collection on adverse events of anti-HIV drugs (D:a:D) risk prediction models. HIV Med. 2016;17(4):289-97.

21. Law MG, Friis-Moller N, El-Sadr WM, Weber R, Reiss P, D'Arminio Monforte A, et al. The use of the Framingham equation to predict myocardial infarctions in HIV-infected patients: comparison with observed events in the D:a:D study. HIV Med. 2006;7(4):218-30.

22. Stamler J, Stamler R, Neaton JD, Wentworth D, Daviglus ML, Garside D, et al. Low risk-factor profile and long-term cardiovascular and noncardiovascular mortality and life expectancy: findings for 5 large cohorts of young adult and middle-aged men and women. JAMA. 1999;282(21):2012-8.

23. Stone NJ, Robinson JG, Lichtenstein AH, Bairey Merz CN, Blum CB, Eckel RH, et al. 2013 ACC/AHA guideline on the treatment of blood cholesterol to reduce atherosclerotic cardiovascular risk in adults: a report of the American College of Cardiology/American Heart Association task force on practice guidelines. Circulation. 2014;129(25 Suppl 2):S1-45.

24. Sauerbrei $W$, Royston $P$, Binder $H$. Selection of important variables and determination of functional form for continuous predictors in multivariable model building. Stat Med. 2007;26(30):5512-28.

25. Luo L, Zeng Y, Li T, Lv W, Wang H, Guo F, et al. Prospective echocardiographic assessment of cardiac structure and function in Chinese persons living with HIV. Clin Infect Dis. 2014;58(10):1459-66.

26. Kuti MA, Adesina OA, Awolude OA, Ogunbosi BO, Fayemiwo SA, Akinyemi JO, et al. Dyslipidemia in ART-naive HIV-infected persons in Nigeria-implications for care. J Int Assoc Providers AIDS Care. 2014;14(4):355-9.

27. Manuthu EM, Joshi MD, Lule GN, Karari E. Prevalence of dyslipidemia and dysglycaemia in HIV infected patients. East Afr Med J. 2008;85(1):10-7. 
28. Tadewos A, Addis Z, Ambachew H, Banerjee S. Prevalence of dyslipidemia among HIV-infected patients using first-line highly active antiretroviral therapy in southern Ethiopia: a cross-sectional comparative group study. AIDS Res Ther. 2012:9(1):31.

29. Armstrong C, Liu E, Okuma J, Spiegelman D, Guerino C, Njelekela M, et al. Dyslipidemia in an HIV-positive antiretroviral treatment-naive population in Dar es salaam, Tanzania. J Acquir Immune Defic Syndr. 2011;57(2):141-5.

30. Shen Y, Wang J, Wang Z, Qi T, Song W, Tang Y, et al. Prevalence of Dyslipidemia among antiretroviral-naive HIV-infected individuals in China. Medicine. 2015;94(48):e2201.

31. Liu J, Hong Y, D'Agostino Sr RB, Wu Z, Wang W, Sun J, et al. Predictive value for the Chinese population of the Framingham CHD risk assessment tool compared with the Chinese multi-provincial cohort study. JAMA. 2004;291(21):2591-9.

32. De Socio GV, Martinelli C, Ricci E, Orofino G, Valsecchi L, Vitiello P, et al. Relations between cardiovascular risk estimates and subclinical atherosclerosis in naive HIV patients: results from the HERMES study. Int J STD AIDS. 2010;21(4):267-72.

33. Rabkin M, Mutiti A, Chung C, Zhang Y, Wei Y, El-Sadr WM. Missed opportunities to address cardiovascular disease risk factors amongst adults attending an urban HIV Clinic in South Africa. PLoS One. 2015;10(10):e0140298.

34. Krikke M, Hoogeveen RC, Hoepelman A, Visseren F, Arends JE. Cardiovascular risk prediction in HIV-infected patients: comparing the Framingham, atherosclerotic cardiovascular disease risk score (ASCVD), systematic coronary risk evaluation for the Netherlands (SCORE-NL) and data collection on adverse events of anti-HIV drugs (D:a:D) risk prediction models. HIV Med. 2016;17(4):289-97.

35. Bonfanti P, De Socio GL, Marconi P, Franzetti M, Martinelli C, Vichi F, et al. Is metabolic syndrome associated to HIV infection per se? Results from the HERMES study. Curr HIV Res. 2010;8(2):165-71.

36. De Socio GV, Ricci E, Parruti G, Calza L, Maggi P, Celesia BM, et al. Statins and aspirin use in HIV-infected people: gap between European AIDS clinical Society guidelines and clinical practice: the results from HIV-HY study. Infection. 2016;44(5):589-97.

37. Boccara F, Miantezila Basilua J, Mary-Krause M, Lang S, Teiger E, Steg PG, et al. Statin therapy and low-density lipoprotein cholesterol reduction in HIVinfected individuals after acute coronary syndrome: results from the PACSHIV lipids substudy. Am Heart J. 2017;183:91-101.

38. De Socio GV, Ricci E, Maggi P, Parruti G, Pucci G, Di Biagio A, et al. Prevalence, awareness, treatment, and control rate of hypertension in HIVinfected patients: the HIV-HY study. Am J Hypertens. 2014;27(2):222-8.

39. Zanni MV, Fitch KV, Feldpausch M, Han A, Lee H, Lu MT, et al. 2013 American College of Cardiology/American Heart Association and 2004 adult treatment Panel III cholesterol guidelines applied to HIV-infected patients with/without subclinical high-risk coronary plaque. AIDS. 2014;28(14):2061-70.

40. Sabin CA, Ryom L, De Wit S, Mocroft A, Phillips AN, Worm SW, et al. Associations between immune depression and cardiovascular events in HIV infection. AIDS. 2013:27(17):2735-48.

\section{Submit your next manuscript to BioMed Central and we will help you at every step:}

- We accept pre-submission inquiries

- Our selector tool helps you to find the most relevant journal

- We provide round the clock customer support

- Convenient online submission

- Thorough peer review

- Inclusion in PubMed and all major indexing services

- Maximum visibility for your research

Submit your manuscript at www.biomedcentral.com/submit 